This is a pre-print of an article published in

Annals of Physics 423 (2020) 168337

The final authenticated version is available online at:

https://doi.org/10.1016/j.aop.2020.168337

\title{
Frequency difference between two clocks at Tokyo Skytree: Contribution of Earth's self-rotation
}

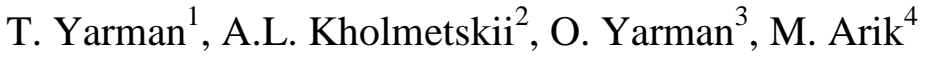 \\ ${ }^{1}$ Department of Engineering, Okan University, Akfirat, Istanbul \\ ${ }^{2}$ Department of Physics, Belarusian State University, 4 Nezavisimosti Avenue, 220030 Minsk, \\ Belarus \\ ${ }^{3}$ Istanbul University, Istanbul, Turkey \\ ${ }^{4}$ Bogazici University, Istanbul, Turkey
}

\begin{abstract}
We address a fundamental issue about the physical meaning of the results of the experiment by M. Takamoto et al. (Nat. Photon. 14, 411 (2020)), where one must emphasize the need to pay attention to the time dilation effect associated with the motion of the clocks around the selfrotational axis of Earth, as seen by a remote observer, not engaged in the rotation of concern. We accentuate the importance of taking into account this effect in the Tokyo Skytree experiment in especially the light of the results gathered from recent measurements of the Mössbauer effect in a rotating system.
\end{abstract}

\section{Introduction}

In the recent paper [1], the authors reported a new and precise test of general relativity (GR) with a pair of optical lattice clocks located at the bottom and top floors of the Tokyo Skytree broadcasting tower and separated by an altitude difference of $\Delta h=452.6$ meters. According to GR, the dependence of the clock rate on the gravitational potential $U$ induces a corresponding frequency shift of light, which, in the approximation of weak gravity, can be presented in the form

$$
\frac{\Delta v}{v}=(1+\alpha) \frac{g \Delta h}{c^{2}}
$$

where $\Delta v=v_{2}-v_{1}$ is the frequency shift in clock 2 located at the top of the tower as compared to clock 1 located on the ground, $g$ is the acceleration of free fall taken at half the tower height, $g \Delta h=\Delta U$ represents the difference between the gravitational potentials of clock 2 and clock 1 respectively, and $c$ stands for the velocity of light in vacuum.

Here, the parameter $\alpha$ is introduced to describe the deviation of the frequency shift (1) from the prediction of GR; for which $\alpha=0$. At this point, it is worth noticing that, nowadays, not only GR, but other theories of gravity too, satisfy the equality $\alpha=0$ in eq. (1) in the case of weak gravity within the achievable measurement precision (see, e.g. [2]). This remark does of course not diminish the significance of developing improved tests of eq. (1), like the one attempted in ref. [1], in order to attain an updated lowest limit for the dimensionless parameter $\alpha$.

We would like to recall that the first experiment on the evaluation of $\alpha$ had been carried out by Pound and Rebka soon after the discovery of the Mössbauer effect [3]. Accordingly, they measured the frequency shift between a resonant source and a resonant absorber with an altitude 
difference of $\Delta h=23 \mathrm{~m}$, where the equality $\alpha<O\left(10^{-2}\right)$ was established. In 2016, a more advanced terrestrial experiment [4], relying on optical lattice clocks separated by $\Delta h=15 \mathrm{~m}$ determined the new limit to be $\alpha=(2.9 \pm 3.6) \times 10^{-3}$.

Contemporaneously, the authors of the recent paper [1] reported

$$
\alpha=(1.4 \pm 9.1) \times 10^{-5}
$$

at $\Delta h=452.6 \mathrm{~m}$ as the lowest limit under terrestrial conditions, which they based on the utilization of very precise optical lattice clocks separated by a considerable distance of about half a kilometer.

Be that as it may, a closer look at the physical and technical background of the Tokyo Skytree experiment outlined in [1] indicates the need to carry out a further analysis in order to draw a final conclusion with respect to the measurement results. Namely, in a sensitive terrestrial experiment proposing to measure the frequency shift of light, one must take into account the fact that the difference of the time rates for the two clocks situated at different altitudes depends not only on the corresponding difference of gravitational potentials according to equation (1), but includes in addition a contribution of the second order Doppler (SOD) effect to the measured frequency shift between said clocks on account of Earth's self-rotation, as seen by a remote observer, not involved with the rotation.

As we will recall in section 2, the SOD contribution to the measured frequency shift is anyway negligible in the experiments [2,3], because the estimated value of this effect lied below the measurement uncertainty and did not affect the estimated limit $\alpha \geq 10^{-3}$. However, this is not the case for the present experiment [1], where the parameter $\alpha$ is claimed to be evaluated at the order of $10^{-4} \ldots 10^{-5}$ (see eq. (2)). As we will show below, the SOD contribution does as a matter of fact affect this parameter already at the order of $10^{-3}$. A way used by the authors of [1] to compensate for this SOD effect is based on the re-definition of $g$ in eq. (1) as the sum of the acceleration of free fall due to gravity and the centripetal acceleration of a terrestrial observer (i.e., effective gravity) due to Earth's self-rotation - which is equivalent to the SOD contribution due to the orbital motion of the clocks from the viewpoint of the outside observer.

Unfortunately, the authors of [1] did not explicitly indicate the physical meaning behind the acceleration $g$ in eq. (1), where, in particular, the parameter $\alpha$ in eq. (1) should, in general, be separated into two parts that are responsible for the contribution of "true gravity" and "effective gravity", correspondingly.

Moreover, we notice that the separate evaluation of the SOD contribution to the measured frequency shift of the clocks at different attitudes acquires an independent significance in the view of the experimental results [5-8], indicating the presence of an additional frequency shift (next to the shift due to the classical dilation of time) in the measurement of the Mössbauer effect in a rotating system between a resonant source and a resonant absorber situated at different radial coordinates. The origin of such an extra energy shift (EES) between the lines of emission and absorption remains the topic of intensive discussions, where a consensus has not been reached up to now (see, e.g., [9-19]). One is therefore inclined to question whether such an EES takes place for the twin clocks at Tokyo Skytree during Earth's self-rotational motion.

In section 2, we elaborate this problem from the viewpoint of a laboratory observer, involved with Earth's self-rotation along with both Tower clocks; where we rely on the Schwarzschild metric to model the gravity of Earth, insofar as specific effects of the Kerr metric [20] remain negligible for terrestrial gravity. Then, we carry out the coordinate transformation from a non-rotating frame (associated with an outside observer) to a rotating frame (associated with a local observer on Earth), and obtain the explicit expression for the difference of the time rate of the clocks at different attitudes both due to gravity and due to Earth's self-rotation.

We further show that the latter component of this difference between the clocks at Tokyo Skytree can be evaluated via the appropriate modification of the measurement algorithm and the data processing of measurement results in the experiment [1].

We discuss and deliver our conclusions in section 3. 


\section{Tokyo Skytree experiment: Analysis and interpretation}

In what follows, we will use the Schwarzschild metric [21]

$$
d s^{2}=\left(1-\frac{2 G M}{r c^{2}}\right) c^{2} d t^{2}-r^{2} \sin ^{2} \vartheta d \varphi^{2}-r^{2} d \vartheta^{2}-\frac{d r^{2}}{1-\frac{2 G M}{r c^{2}}}
$$

for the description of Earth's gravity from the viewpoint of a distant observer. Here $M$ is the mass of the Earth, $G$ is the gravitational constant, $c$ is the light velocity in vacuum, and $r, \vartheta, \varphi$ are the spherical coordinates.

In order to determine the corresponding metric expression for an observer co-rotating with the Earth, we carry out the transformation of spherical coordinates in the case of planar rotation:

$$
r^{\prime}=r, \vartheta^{\prime}=\vartheta, \varphi^{\prime}=\varphi+\omega t, t^{\prime}=t
$$

Here, we neglected the inclination of the rotational axis of Earth, which is a warranted approximation in the subsequent analysis of the tiny effects of time dilation.

Thus, substituting (4) into (3), we obtain for an observer on the surface of the Earth:

$$
\begin{aligned}
& d s^{\prime 2}=c^{2} d t^{\prime 2}\left(\left(1-\frac{2 G M}{r c^{2}}\right)-\frac{\omega^{2} r^{\prime 2} \sin ^{2} \vartheta^{\prime}}{c^{2}}\right)-\frac{d r^{\prime 2}}{1-\frac{2 G M}{r c^{2}}}- \\
& r^{\prime 2} \sin ^{2} \vartheta^{\prime} d \varphi^{\prime 2}-r^{\prime 2} d \vartheta^{\prime 2}+2 \omega r^{\prime 2} \sin ^{2} \vartheta^{\prime} d \varphi^{\prime} d t^{\prime} .
\end{aligned}
$$

Eq. (5) shows that the metric coefficient

$$
g_{00}=c^{2} d t^{\prime 2}\left(\left(1-\frac{2 G M}{r c^{2}}\right)-\frac{\omega^{2} r^{\prime 2} \sin ^{2} \vartheta^{\prime}}{c^{2}}\right),
$$

so that the proper time in any spatial point on this frame is defined by the relationship

$$
d \tau^{\prime}=\frac{1}{c} \sqrt{g_{00}} d t^{\prime}=d t^{\prime}\left(\left(1-\frac{2 G M}{r c^{2}}\right)-\frac{\omega^{2} r^{\prime 2} \sin ^{2} \vartheta^{\prime}}{c^{2}}\right)^{1 / 2} .
$$

To a sufficient accuracy of calculations at $c^{-2}$, we have

$$
d \tau^{\prime} \approx d t^{\prime}\left(1-\frac{G M}{r c^{2}}-\frac{\omega^{2} r^{\prime 2} \sin ^{2} \vartheta^{\prime}}{2 c^{2}}\right) .
$$

Applying eq. (8) to the Tokyo Skytree experiment, we adopt $r_{\text {low }}=R$ for the ground level clock, and $r_{u p}=R+\Delta h$ for the top floor clock, where $R$ is the radius of the Earth. Taking also into account that the measured frequency is inversely proportional to the time rate, we obtain:

$$
v(R+\Delta h)-v(R) \approx v_{0}\left(\frac{G M \Delta h}{R^{2} c^{2}}-\frac{\omega^{2} R \Delta h \cos ^{2} \vartheta}{2 c^{2}}\right) .
$$

Further on, denoting the acceleration of free fall on the surface of the Earth due to its gravity as

$$
\frac{G M}{R^{2}}=g_{\text {gravity }},
$$

and re-defining straightforwardly the experimental parameter $\alpha$ of eq. (1) in a way where it becomes relevant only for the true gravity of Earth, we obtain for the relative variation in the frequency of the clocks between the top and bottom floors of the Tokyo Skytree:

$$
\left(\frac{\Delta v}{v}\right)_{\text {total }}=(1+\alpha) \frac{g_{\text {gravity }} \Delta h}{c^{2}}-\frac{\omega^{2} R \Delta h \cos ^{2} \vartheta}{c^{2}} .
$$

Thus, the total frequency shift contains two substantially contributing terms: The component 


$$
\left(\frac{\Delta v}{v}\right)_{\text {gravity }}=(1+\alpha) \frac{g_{\text {gravity }} \Delta h}{c^{2}}
$$

due to Earth's gravity, and the component

$$
\left(\frac{\Delta v}{v}\right)_{S O D}=-\frac{\omega^{2} R \Delta h \cos ^{2} \vartheta}{c^{2}}
$$

due to the effective gravitational potential responsible for the centrifugal force in the frame corotating with the Earth. For an outside observer not involved with Earth's self-rotation, the contribution (12b) ensues from the difference of the orbital velocities of both clocks rotating along with Earth at different radii $R$ and $R+h$, respectively. This leads to the corresponding difference of frequencies due to the second order Doppler (SOD) effect, which, to the adopted accuracy of calculations at $c^{-2}$, is given by eq. (12b).

At this point, it is worth noticing that Yarman et al. had already laid out a problem identical to the present one, and derived the above result via a different route [22].

At any rate, the contribution (12b) could not be explicitly extracted from any of the previous terrestrial experiments aimed at measuring the influence of gravity on the frequency shift of electromagnetic radiation (see, e.g., $[3,4]$ ) - because i) the altitude difference $\Delta h$ between the two clocks was at least one order of magnitude smaller than that exercised in the experiment [1] and ii) the measurement uncertainty with respect to the frequency shift of electromagnetic radiation was two-three orders of magnitude larger in comparison with the experiment [1]. Contrariwise, with regards to the experiment [1], the SOD contribution (2) could and ought to have rather been explicitly framed as such. Indeed, substituting into eq. (12b) the corresponding numerical values from [1] $\left(\omega=2 \pi / 86164.1=0.7292 \times 10^{-4}, R=6.375 \times 10^{6} \mathrm{~m}, \vartheta=35.71^{\circ}\right.$ for Tokyo, $\Delta h=452.596$ $\mathrm{m}$ for the Skytree tower setup), we obtain

$$
\left(\frac{\Delta v}{v}\right)_{S O D}=-1.13 \times 10^{-16}
$$

which thus remains two orders of magnitude above the relative measurement uncertainty in the frequency shift that is estimated to be at the order of $10^{-18}$ in [1]. It means, in particular, that - in the precise determination of the parameter $\alpha$ - equation (1) should be replaced by equation (11). This observation requires the authors' new lowest limit claim (2) with regards to the parameter $\alpha$ in eq. (1) to be recalibrated.

A possible way to incorporate the SOD contribution (12b) into the measured frequency shift is to measure the acceleration of the free fall $g$ via the balance of gravitational and elastic forces acting on a test mass. In this way - as realized in the experiment [1] with the application of LaCoste \& Romberg (model G) and Scintext CG-5 Autograv gravimeters - the balance of forces on a test mass is sensitive not only to the proper value of the gravitational force, but additionally gets affected by the centrifugal force acting on the test mass due to Earth's self-rotation. Therefore, the component of the static balancing force $F$ along the normal to the Earth's surface should rather be defined by the equality

$$
F=m g_{\text {measured }}=m g_{\text {gravity }}-m \omega^{2} R \cos ^{2} \vartheta .
$$

Here, the value which we denoted as $g_{\text {measured }}$ in eq. (14) has, in fact, been used for calculations in the experiment [1]; whereas, in a sophisticated approach, the influence of gravity to the measured frequency shift should have been defined via the true acceleration of the free fall (10) - i.e., $U=g_{\text {gravity }} \Delta h$. Then, using eq. (14), we can present the total frequency shift in the form

$$
\begin{aligned}
& \left(\frac{\Delta \mathrm{v}}{\mathrm{v}}\right)_{\text {total }}=\left(\frac{\Delta \mathrm{v}}{\mathrm{v}}\right)_{\text {gravity }}+\left(\frac{\Delta \mathrm{v}}{\mathrm{v}}\right)_{S O D}=(1+\alpha) \frac{g_{\text {gravity }} \Delta h}{c^{2}}-\frac{\omega^{2} R \Delta h \cos ^{2} \vartheta}{c^{2}}= \\
& (1+\alpha) \frac{g_{\text {measured }} \Delta h}{c^{2}}+\alpha \frac{\omega^{2} R \Delta h \cos ^{2} \vartheta}{c^{2}} \approx(1+\alpha) \frac{g_{\text {measured }} \Delta h}{c^{2}},
\end{aligned}
$$


which explicitly demonstrates that, in eq. (1) used by the authors [1], the acceleration $g$ is to be understood as $g_{\text {measured }}$ the way defined by (14).

At the same time, the disclosed physical mechanism responsible for the observed frequency shift of electromagnetic radiation at different altitudes given by eq. (15) allows us to formulate one more crucial experimental problem aimed at the precise measurement of the component of the frequency shift for two clocks, related to their rotational motion around the selfrotational axis of Earth, at the bottom and at the top of the Tokyo Skytree.

The growing interest shown towards this problem is related to a recent disclosure regarding the extra energy shift (next to the classical SOD shift) between emitted and absorbed resonant Mössbauer radiation as first revealed in ref. [6] via the re-analysis of the familiar experiment by Kündig [5] - which was later confirmed by modern Mössbauer experiments in a rotating system $[7,8]$.

At the moment, there are various attempts to explain the origin of said extra-energy shift (EES) (see, e.g. [9-14]), though the majority cannot be recognized as acceptable (see, e.g., [1519]). Among them, the so-far only successful explanation [9] is based on the quantum mechanical behavior of resonant nuclei confined in crystal cells. Therefore, for optical lattice clocks involved in motion along Earth's axis of self-rotation, such an EES will not emerge if the explanation under [9] is correct, and this conjecture can be checked via the re-analysis of the data from the Tokyo Skytree experiment.

In formal terms, any possibility for the presence of the EES in the experiment [1] can be described via the modification of eq. (12b) into

$$
\left(\frac{\Delta v}{v}\right)_{S O D}=-2 k \frac{\omega^{2} R \Delta h \cos ^{2} \vartheta}{c^{2}}
$$

where the newly introduced coefficient $k$ is equal to $1 / 2$ in cases where no EES takes place, and $k>0.5$, if EES is present.

Using eq. (16), we can modify eq. (11) to the form

$$
\left(\frac{\Delta v}{v}\right)_{\text {total }}=\left(\frac{\Delta v}{v}\right)_{\text {gravity }}+\left(\frac{\Delta v}{v}\right)_{S O D}=(1+\alpha) \frac{g_{\text {gravity }} \Delta h}{c^{2}}-2 k \frac{\omega^{2} R \Delta h \cos ^{2} \vartheta}{c^{2}} \text {. }
$$

Eq. (17) indicates that, for a precise measurement of $k$, one has to carry out a direct measurement of $g_{\text {gravity }}$ instead of the determination of $g_{\text {measured }}$, as has been done in the experiment [1].

Such a direct measurement of $g_{\text {gravity }}$ in eq. (17) can be realized with, e.g., a precise ballistic gravimeter based on the measurement of the duration of the free fall of a test mass.

In such a way, we can re-direct the goal of the Tokyo Skytree experiment from the estimation of the parameter $\alpha$ in eq. (1) to the measurement of the parameter $k$ in eq. (17), given that, in any reasonable theory of gravity, $\alpha$ should be anyway equal to zero. At the same time, the evaluation of the numerical value of the coefficient $k$ in eq. (17) will allow us to answer the question as to whether or not the EES is specific to Mössbauer rotor experiments where the quantum mechanical behavior of resonant nuclei must be taken into account.

Assuming that the accuracy of measurement with respect to the free fall acceleration $g_{\text {gravity }}$ via the ballistic method is comparable with the accuracy of measurement with respect to $g_{\text {measured }}$ via the balance of gravitational and elastic forces on a test mass as relied on in [1], one can straightforwardly find that the relative measurement uncertainty in the evaluation of the coefficient $k$ via equation (17) will be less than $10 \%$ under the conditions of the Tokyo Skytree experiment.

\section{Conclusion}

We have shown that the outstandingly high measurement accuracy achieved in the experiment [1] with respect to the frequency shift of electromagnetic radiation at different attitudes requires one to consider not only the gravitational shift component (delineated by eq. (12a) above) - as it 
was deemed sufficient in all previous terrestrial experiments on the subject (e.g., [3, 4]) - but additionally the frequency shift (12b) resulting from the tangential motion of clocks involved in Earth's self-rotation as seen by a remote (non-rotating) observer.

Under these conditions, we have pointed out the importance of the direct measurement of the acceleration of free fall on the surface of Earth due to its gravity alone $\left(g_{\text {gravity }}\right)$, with the exclusion of the centrifugal acceleration caused by Earth's self-rotation.

This way will allow us to apply a more sophisticated algorithm with regards to the processing of the experiment [1], through the explicit evaluation of the frequency shift component due to the rotational motion of the clocks from the viewpoint of an outside observer (i.e., the SOD effect) according to eq. (16).

We would like to emphasize that the verification of eq. (17) for the total frequency shift between two clocks - via the measurement of $g_{\text {gravity }}$ and with the adoption of the equality $\alpha=0-$ will allow evaluating the numerical value of the coefficient $k$, which is highly topical in the light of recent Mössbauer experiments in a rotating system where the origin of the EES between emitted and absorbed resonant radiation remains the subject of intense discussions at present.

Finally, it would be relevant to recall that Yarman et al. [22] had already posed and solved a problem identical to the Tokyo Skytree experiment about a decade ago, inasmuch as predicting $k=1 / 2$ - as also follows from the standard treatment of the same problem under GR via comparing eqs. (12b) and (16).

\section{References}

1. M. Takamoto et al., Nat. Photon. 14, 411 (2020).

2. C.M. Will, Living. Rev. Relat. 17, 4 (2014).

3. R.V. Pound, G.A. Rebka Jr., Phys. Rev. Lett. 3, 439 (1959).

4. T. Takano et al., Nat. Photon. 10, 662 (2016).

5. W. Kündig, Phys. Rev. 129, 2371 (1963).

6. A.L. Kholmetskii, T. Yarman, O.V. Missevitch, Phys. Scr. 78, 035302 (2008).

7. A.L. Kholmetskii, T. Yarman, O.V. Missevitch, B.I. Rogozev, Phys. Scr. 79, 065007 (2009).

8. T. Yarman, et al., Can. J. Phys. 94, 780 (2016).

9. T. Yarman, A.L. Kholmetskii, M. Arik, Eur. Phys. J. Plus 130, 191 (2015).

10. Y. Friedman et al., EPL 114, 50010 (2016).

11. E. Benedetto, A. Feoli, Eur. Phys. J. Plus 133, 53 (2018).

12. C. Corda, Int. J. Mod. Phys. D 28, 1950131 (2019).

13. G. Iovane, E. Benedetto, Ann. Phys. 403, 106 (2019).

14. S.A. Podosenov, J. Foukzon, E.R. Men'kova, Ann. Phys. 413, 168047 (2020).

15. A.L. Kholmetskii, T. Yarman, O. Yarman, M. Arik, Eur. Phys. J. Plus 133, 261 (2018).

16. A.L. Kholmetskii, T. Yarman, O. Yarman, M. Arik, Int. J. Mod. Phys. D 28, 1950127 (2019).

17. A.L. Kholmetskii, T. Yarman, O. Yarman, M. Arik, Ann. Phys. 409, 167931 (2019).

18. A.L. Kholmetskii, T. Yarman, O. Yarman, M. Arik, Ann. Phys. 411, 167912 (2019).

19. A.L. Kholmetskii, T. Yarman, O. Yarman, M. Arik, Ann. Phys. 418, 168191 (2020).

20. G.C. Debney, R.P. Kerr, A. Schild, J. Math. Phys. 10, 1842 (1969).

21. L. Landau, E. Lifshitz, Classical Theory of Fields, 3rd ed. (London, Pergamon 1971).

22. T. Yarman, M. Arik, A.L. Kholmetskii, TWMS J. App. Eng. Math. 1, 109, 2011. 